\title{
A New Scheme for Multisensor Image Matching
}

\author{
Hailong Wang and Shuqin Zhang \\ School of Computer, Zhongyuan University of Technology, Zheng Zhou, China \\ whlseadragon@163.com, zsqheu@163.com
}

Keywords: Iteration; Projective transformation; Affine transformation; Nonlinear representation

\begin{abstract}
A new iteration scheme for infrared and visible image matching is presented in this paper. The goal is to derive a new method for highly accurate matching of multisensor images. The existing matching algorithms based on image pyramid decomposition have been popular as they have advantages of low computational complexity. However they can only be applied to images from the same sensor. The proposed new iteration approach can register both visible and infrared images. To capture large displacements, coarse-to-fine new refinement of the matching parameters is performed based on the Gaussian pyramid. Parameters are propagated on each round of iteration from a low level to a higher resolution to produce more accurate parameters. Experimental results for matching of infrared and visible images show that the proposed scheme is effective and reliable.
\end{abstract}

\section{Introduction}

Image matching is the process of aligning two images of the same scene so that corresponding points in the scene are placed in identical pixel positions. It is an important component of image fusion. It is necessary to ensure that images to be fused are registered so that they can be compared and combined. Automatic matching of images obtained from multiple sensors is a difficult task because of the mismatch in the images caused by local polarity reversals and complementary features. For remote sensing, matching of infrared to visible spectra is particularly important for measuring, detecting, and tracking regions of interest.

Existing matching algorithms fall into two basic categories: feature-based and blind. Featured-based matching attempts to identify edges, corner points, contours or other features that are common to both images, and then uses standard geometric transforms to obtain mapping between the two [1,2]. However features are subjected to temperature variations and edges in an infrared image may not be present in visible images. Likewise, visible features may disappear in the infrared spectrum. Consequently, feature-based matching is mainly used for locating features common to both images.

The second category of approaches registers images blindly. Typical matching methods are to minimize the sum of squared differences of pixel values or to maximize the normalized correlation of the images [3]. Fourier-based techniques, phase correlation, and mutual information are suited for multisensor images. But they usually have high computational complexity. A novel pyramidal scheme based on Laplacian or Gaussian decomposition is proposed in this paper, which can reduce the computational complexity.

\section{Matching Algorithm}

Motion between images can be described as follows:

Associate a motion vector $(\mathrm{p} \mathrm{x}, \mathrm{p} y)$ with each pixel $(\mathrm{x}, \mathrm{y})$ in image $\mathrm{I}_{1}$ to be aligned with image $\mathrm{I}_{2}$. Formulate the motion as $\left(p_{x}, p_{y}\right)=f(x, y ; P)$, where $P$ is a parameter vector that is identical within the entire image, and the function $\mathrm{f}$ determines the structure of the motion at each pixel. This is a model of global parametric motion. 
For planar scenes, it is advantageous to use global parametric motion model [4 6]. If $I_{1}$ and $I_{2}$ are the two images to be registered, then $\mathrm{I}_{2}(\mathrm{x}, \mathrm{y})$ can be expressed in terms of $\mathrm{I}_{1}(\mathrm{x}, \mathrm{y})$ :

$$
I_{2}(x, y)=I_{1}\left(x+p_{x}, y+p_{y}\right)
$$

Where $p_{\mathrm{x}}(\mathrm{x}, \mathrm{y}: \mathrm{P})$ and $p_{\mathrm{y}}(\mathrm{x}, \mathrm{y}: \mathrm{P})$ depend on $(\mathrm{x}, \mathrm{y})$, the parameters $P$ and the model are used to describe the global motion. Provided that the scene is a planar surface, motion of the scene can be approximated by a projective transformation [2]:

$$
\begin{aligned}
& p_{x}(x, y, P)=p_{1}+p_{3} x+p_{5} y+p_{7} x^{2}+p_{8} x y \\
& p_{y}(x, y, P)=p_{2}+p_{4} x+p_{6} y+p_{7} x y+p_{8} y^{2}
\end{aligned}
$$

The matching parameters $\mathrm{P} \equiv\left[\mathrm{p}_{1}, \mathrm{p}_{8}\right]$ are the globe motion model. When distance between the scene and the camera is large, the motion can be approximated by an affine model with $\mathrm{p}_{7}=\mathrm{p}_{8}=0$ in Eqs. (2) and (3). Translation is a special case of the projective model when $p_{3}=p_{4}=p_{5}=p_{6}=p_{7}=p_{8}=0$ in Eqs. (2) and (3). $P$ is estimated by minimizing the sum of squared error:

$$
E=\sum_{x, y}\left(I_{2}(x, y)-I_{1}\left(x+p_{x}, y+p_{y}\right)\right)^{2}
$$

If displacements ( $\mathrm{p} x, \mathrm{p} y$ ) are small, the above equation can be simplified with a Taylor series approximation:

$$
I_{1}\left(x+p_{x}, y+p_{y}\right)=I_{1}(x, y)+p_{x} \frac{\partial I_{1}}{\partial x}+p_{y} \frac{\partial I_{1}}{\partial y}
$$

The sum of squared error can be expressed as:

$$
E=\sum_{x, y}\left(I_{2}(x, y)-I_{1}(x, y)-p_{x} \frac{\partial I_{1}}{\partial x}-p_{y} \frac{\partial I_{1}}{\partial y}\right)^{2}
$$

Motion between the images is obtained by setting the derivatives of the error measure in Equation (6) with respect to the parameters of motion model to zero and solving the resulting system of equations. This, however, holds only when displacements are small. To capture large displacements, here we propose a coarse to fine new refinement of the matching parameters in a multiresolution framework based on Laplacian or Gaussian decomposition.

The matching parameters are first iteratively estimated at a lower resolution to obtain a coarse solution. The solution is then used to initialize the parameter estimation at a higher resolution to obtain more accurate parameters. Features are used to selected matching control points.

Features such as contours, edges, corners, and lines in one image are matched to those in the other. Control points are manually selected to match the two images. Matching is performed by finding a geometric mapping that aligns these control points. A set of four match points $\left(\mathrm{x}_{\mathrm{i}}, \mathrm{y}_{\mathrm{i}}\right)$ and, $\mathrm{i}=1,2,3,4$, in images I1 and I2 provide a system of eight simultaneous equations in terms of the unknown parameters $\mathrm{P} \equiv\left[\mathrm{p}_{1}, \ldots, \mathrm{p}_{8}\right]$, the equation are solved to obtain estimates of the parameters, which are used to align the images. The scheme is illustrated in Fig. 1. In the block diagram, R represent refining control points, and $\mathrm{P}$ the propagating parameters. Steps of the matching are as follows:

The two images, A1 and B1, to be registered are transformed into 3 levels: A1, A2, A3; B1, B2, and B3 of a Laplacian pyramid.

Each level is then passed through a low-pass filter as described above, the resulting representation is fed into the parameter estimation step.

Matching proceeds newly from lower resolutions (higher levels of the pyramid) to higher resolutions 
(lower levels of the pyramid).

On each level, refining control points of the same feature are selected in the images, and the parameters are iteratively estimated by minimizing the error measure. Parameters estimated at a lower resolution are used as an initial guess at a higher resolution.

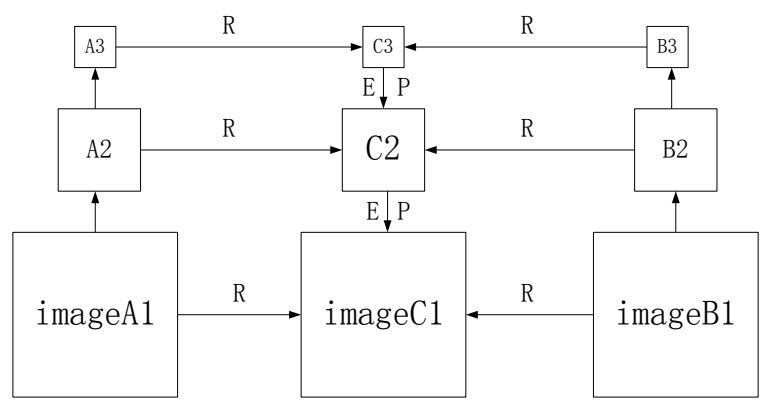

Figure 1. Block diagram of matching process.

The parameters are propagated from one level to another with a change in coordinates, and the resolution is doubled in size. The parameters $\mathrm{p}_{1}$ and $\mathrm{p}_{2}$ are multiplied by 2 , and $\mathrm{p}_{7}$ and $\mathrm{p}_{8}$ are divided by 2. All other parameters remain the unchanged.

\section{Experiments and the Results}

First, we apply the described approach to pairs of images obtained with the same sensor, Fig. 2(a) and 2(b) show a pair of visible images that are not registered. Fig. 2(c) is the aligned result.

Secondly, the algorithm is applied to register several sets of multisensor images. Fig. 3(a) and 3(b) are images of the Pentagon obtained from sensors operating in different portions of the infrared spectrum. These images have been registered using the projective model. Fig. 3(c) shows the superimposed images after being registered. The result shows that the edges of structures and the roads in the two images are properly aligned. Fig. 4(a) and 4(b) are visible band and IR images, respectively, and show a runway scene as an aircraft is about to land. These images contain local polarity reserved features as well as overall gray level disparities. Fig. 4(c) shows the matching, from the result the edges of the runway and the taxiways are now aligned.

The proposed approach is evaluated by using some simulated multisensor images in which the correct matching is known. The experiments have indicated the validity of the scheme

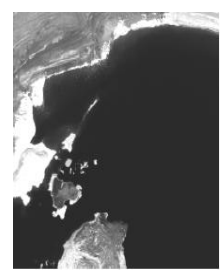

2(a)

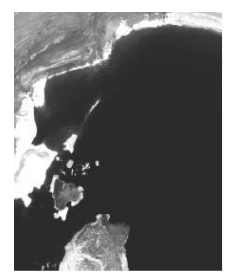

2(b)

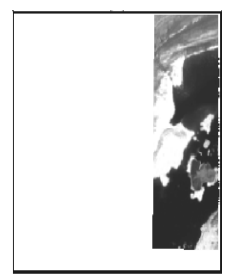

2(c)

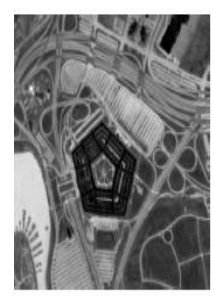

3(a)

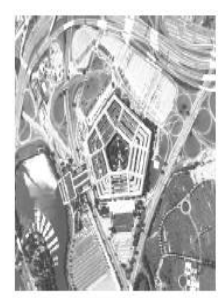

3(b)

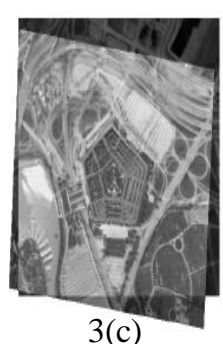

3(c)

Figure 2. The process of the matching for images of the same sensor

Figure 3. Matching of Infrared image and visible image of the Pentagon 


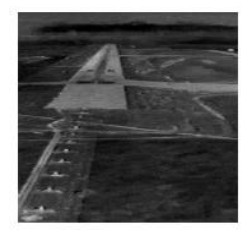

4(a)

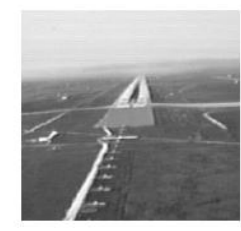

4(b)

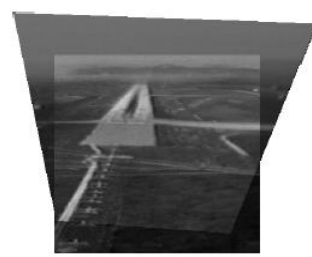

$4(c)$

Figure 4. Showing the visible band and IR image of

\section{Discussion and Remarks}

the airport runway

We have described a new approach for matching of multisensor images. It provides a solution to the problem that arises in the case of multiple sensors. The traditional matching techniques are either unable to find the same feature existing in different images or too complex in computation. We use the control points to register images by using pyramidal decomposition based on coarse-to-fine iteration.

The matching algorithm can incorporate some prior knowledge about locations of certain features to improve the alignment. It is important to note that knowledge of local polarity reserved and the complementary features can aid the task of alignment.

It is imperative to register images before performing data fusion, therefore matching itself can be considered a form of fusion as it involves comparison and combination of information in images. The discussion on matching has been focused on spatial misalignments of the image features. The experiments show that the proposed scheme is valid.

\section{Acknowledgment}

Financial supports from China Shan xi Technology Program are highly appreciated. The helpful comments from two anonymous reviewers are also gratefully acknowledged.

\section{References}

[1] V. Govindu, C. Schekhar, Alignment using distribution of local geometric properties. IEEE Trans. on PAMI, 1999, 21(10): 1031-1034.

[2] X.Dai, S.Khorram, Development of a feature-based approach to automated image matching for multitemporal and multisensor remotely sensed imagery. Proc 1997 IGARSS, Remote Sensing-A Scientific Vision for Sustainable Development, 1997, 1(8):243-245.

[3] B. Dasgupta, B. N. Chatterji, Fourier Mellin transform based image matching algorithm, J. Inst. Electron. Telecommun. Eng. 1996, 42(1): 3-9.

[4] C. D. Kuglin, D. C. Hines, The phase correlation image alignment method. IEEE Conf. on Cybernetics and Society, Sept, 1975, pp.163-165.

[5] B. Srinivasa Reddy, B. N. Chatterji. An FFT-Based Technique for translation, rotation, and scale invariant image matching. IEEE Trans. Image Proce ssing. 1996, 5(8): 1266-1271.

[6] Harold S. Stone, Robert Wolpov. Blind cross spectral image matching using prefiltering and Fourier-based translation detection. IEEE Trans. on Geoscience and Remote Sensing. 2002, 40(3): 637-650.

[7] Wei Song, Jiaqi Zhang. Based on the ViBe of substation intelligent monitoring technology research [J]. Journal of instruments and meters, 2014, S2:36-41. 
[8] Lidan, Zhaojia, Shanshan zhou, Zhou Wei. A point target detection algorithm based on adaptive background partition [J]. Infrared technology, 2011, 01:32-40.

[9] HuaYuanLei, Wan-jun Liu. The improvement of the Gaussian mixture model moving target detection algorithm [J]. Journal of computer applications, 2014, 11:580-584.

[10] Guo wei, Liu Xinyan XiaoZhenJiu. Target detection based on improved Gaussian mixture model [J]. Journal of computer applications and software, 2015, 11:148-166. 